\title{
Manifold Learning for Image-Based Breathing Gating with Application to 4D Ultrasound*
}

\author{
Christian Wachinger, Mehmet Yigitsoy, and Nassir Navab \\ Computer Aided Medical Procedures (CAMP), TUM, Munich, Germany \\ \{wachinge, yigitsoy, navab\}@cs.tum.edu
}

\begin{abstract}
Breathing motion leads to a significant displacement and deformation of organs in the abdominal region. This makes the detection of the breathing phase for numerous applications necessary. We propose a new, purely image-based respiratory gating method for ultrasound. Further, we use this technique to provide a solution for breathing affected 4D ultrasound acquisitions with a wobbler probe. We achieve the gating with Laplacian eigenmaps, a manifold learning technique, to determine the low-dimensional manifold embedded in the high-dimensional image space. Since Laplacian eigenmaps assign each ultrasound frame a coordinate in low-dimensional space by respecting the neighborhood relationship, they are well suited for analyzing the breathing cycle. For the $4 \mathrm{D}$ application, we perform the manifold learning for each angle, and consecutively, align all the local curves and perform a curve fitting to achieve a globally consistent breathing signal. We performed the imagebased gating on several 2D and 3D ultrasound datasets over time, and quantified its very good performance by comparing it to measurements from an external gating system.
\end{abstract}

\section{Introduction}

Imaging organs in thorax and abdomen is affected by respiratory motion. For consecutive processing steps, it is often necessary to assign to each image its corresponding breathing phase. This is achieved with external gating devices, which the patient gets connected to. These devices, however, have long setup times, prolong the overall acquisition, are costly, and consequently, rarely used in practice. Moreover, the synchronization of image data and breathing signal is not trivial. While certain imaging devices, such as CT and MR scanners, support the connection of respiratory gating systems, we are not aware of such a possibility for ultrasound; leaving the synchronization to the user. We propose an image-based respiratory gating system for ultrasound using manifold learning. Moreover, we use this technique to provide a solution for acquiring breathing affected 4D ultrasound with a wobbler probe. The proposed method is fully automatic, and does not need any prior information or training data.

\footnotetext{
* This work was partly funded by the European Commission. We want to thank Athanasios Karamalis and Oliver Kutter for discussions.
} 
$\underline{\text { Wobbler }}$

Breathing
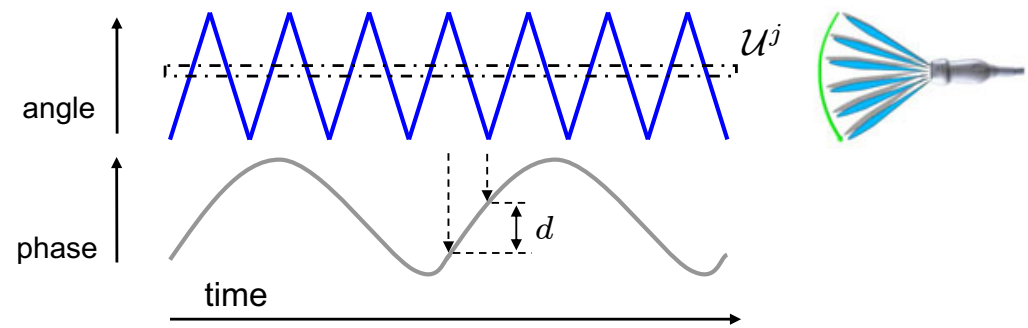

Fig. 1. Wobbler angle (blue) and respiratory phase (gray) over time. Dashed lines indicate respiratory change $d$ within one sweep. Dash dotted line indicates frames from same angle over several breathing cycles.

One of the applications for which the assignment of the respiratory phase is important is $2 \mathrm{D}$ and $3 \mathrm{D}$ ultrasound mosaicing. In order to achieve good results, images from the same respiratory phase have to be combined. An alternative to breathing gating are breath-hold acquisitions, but they further complicate the procedure and are dependent on the patients ability for breath-hold. Another application that we investigate in more details throughout the article, and for which we have not yet found a solution proposed in the literature, is the acquisition of breathing affected 4D ultrasound with a mechanically steered transducer, also referred to as wobbler. The problems for using a wobbler in such a scenario is that images in one sweep do not contain consistent information, but represent the anatomy in different breathing phases. We illustrate this in figure 1 , where we schematically plot the deviation angle of the wobbler together with the respiratory signal over time. The phase difference $d$ indicates the range of breathing phases accumulated in one sweep. We propose to select all frames acquired from the same angle (dash dotted line) and apply the image-based gating on each of those sets of images. Having the respiratory signal estimated for each angle, we align these curves and apply a robust spline curve fitting to create a globally consistent respiratory signal. This, consequently, allows us to reconstruct volumes for specific breathing stages. An alternative to the application of a wobbler would be a native $3 \mathrm{D}$ transducer with elements arranged on a $2 \mathrm{D}$ array. Those systems, however, are still expensive and the access to their data streaming and radio frequency data is very restricted.

\subsection{Related Work}

There are several papers on image-based gating in ultrasound for detecting the cardiac motion [1/234]. These approaches apply techniques that are either (i) specific to detecting the cardiac signal e.g. centroid algorithm [4, (ii) based on user interaction [1], or (iii) designed for intravascular ultrasound [2]3]. We are only aware of the work of Sundar et al. [5], where an automatic technique for breathing gating in ultrasound is proposed. It bases on the phase correlation technique to estimate the motion between successive frames. The breathing phase is estimated from the energy change between consecutive frames. The inherent 
limitation of the phase correlation algorithm is that it finds the global translation in the image plane. Considering the case of $2 \mathrm{D}$ ultrasound, the organ motion is not necessarily in-plane, and consequently, there is no uniform global translation.

Several manifold learning techniques were proposed in the literature with common techniques being Isomap [6] and Laplacian eigenmaps [7. Since its introduction, manifold learning has been applied for a multitude of applications, including segmentation [8], registration [9], tracking [10], recognition [1], and 4D CT reconstruction [12]. The approach taken in the reconstruction is similar to ours because Isomap is used to estimate the breathing phase on CT slabs. In our work, we deal with the specific challenges of the integration of 4D ultrasound wobbler data. To this end, we focus on Laplacian eigenamps, since we achieved better results in comparison to Isomap.

\section{Method}

The general idea of manifold learning is to project a manifold in high dimensional space $\mathbb{R}^{N}$ to a low dimensional space $\mathbb{R}^{n}$, while preserving the local neighborhood. For our application, we consider one dimension of the ambient space for each image pixel, so $N$ is corresponding to the resolution of the ultrasound images. For the low dimensional space, we set $n=1$, because we want to use the coordinate of the points directly as breathing phase estimation. Considering $k$ ultrasound images $\mathcal{U}=\left\{\mathbf{u}_{1}, \ldots, \mathbf{u}_{k}\right\}$ that are acquired over several breathing cycles, the manifold learning $m$ assigns each image a coordinate in the low dimensional space $\phi_{i}$

$$
\begin{aligned}
m: \mathbb{R}^{N} & \rightarrow \mathbb{R}^{1} \\
\mathbf{u}_{i} & \mapsto \phi_{i},
\end{aligned}
$$

with $1 \leq i \leq k$. The suggestion that ultrasound images lie on a low dimensional manifold in the ambient space seems to be justified because variations between neighboring slices are smooth, and further, slices from the same respiratory phase but different acquisition times are similar. Moreover, since manifold learning techniques try to optimally preserve local information [7], meaning that similar images are mapped to similar positions in the low dimensional space, it is reasonable to use $\phi_{i}$ as an estimate for the respiratory phase.

\subsection{Laplacian Eigenmaps}

We propose the application of Laplacian eigenmaps [7] for the respiratory phase estimation because the technique is well founded on mathematical concepts (Laplace Beltrami operator) and computationally efficient. Laplacian eigenmaps build upon the construction of a graph, which represents the neighborhood information of the data set. Subsequently, the graph Laplacian is applied to calculate a low-dimensional representation of the data that preserves the local neighborhood information in an optimal way. 


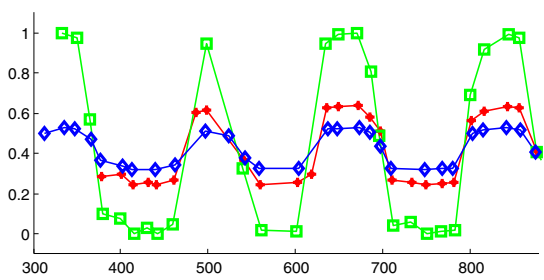

(a) Not Aligned

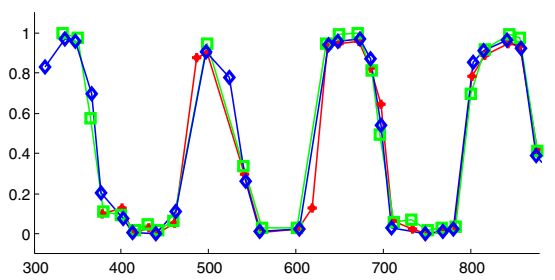

(b) Aligned

Fig. 2. Breathing signals from manifold learning. Illustrated is the case for 3 angles (3 colors). X-axis indicates ultrasound frame number. The plots show the signals before and after alignment.

We construct a graph with a node for each point $\mathbf{u}_{i}$ and with edges connecting neighboring nodes. We select for each image $\mathbf{u}_{i}$ the $l$ nearest neighbors, by evaluating the norm $\left\|\mathbf{u}_{i}-\mathbf{u}_{\iota}\right\|^{2}$. Further, heat kernel-based weights are assigned to the edges with $w_{i \iota}=e^{-\left\|\mathbf{u}_{i}-\mathbf{u}_{\iota}\right\|^{2} /\left(2 \cdot \sigma^{2}\right)}$ and $\sigma^{2}$ the variance [7. The similarity measure is important for neighborhood selection and weighting, where the calculation of the Euclidean norm between the points is equivalent to calculating the sum of squared differences (SSD) between the images. A vast number of similarity measures is proposed in the context of medical image registration. Since we deal with monomodal data for our application, we investigate the performance of SSD and the correlation coefficient (CC). The calculation of CC is similar to the calculation of SSD on normalized input images. Once the neighborhood graph is constructed, the eigenvectors of the graph Laplacian provide the embedding map.

\subsection{Global Consistency}

After the breathing gating is performed for each angle, we have to establish the correspondence between different angles in order to construct the global respiratory signal. Be $\mathcal{U}$ the set of all acquired images. We partition the set in disjunct subsets $\mathcal{U}^{1}, \ldots, \mathcal{U}^{\alpha}$, corresponding to the number of different deflection angles $\alpha$ of the wobbler (dash dotted region in figure1). We perform the manifold learning for each of the subsets separately $m^{j}\left(\mathbf{u}_{i}\right)=\phi_{i}^{j}$, with $1 \leq j \leq \alpha$. So depending on the acquisition angle of the ultrasound image $\mathbf{u}_{i}$, the corresponding manifold learning $m^{j}$ is performed. Considering all the phases estimated from one angle, we have the local respiratory signals $\Phi^{j}=\left\{\phi_{1}^{j}, \ldots, \phi_{v}^{j}\right\}$, with $v$ the number of frames per angle. Each local signal contains a consistent estimation of the breathing signal. It is, however, not possible to directly compare local signals, because the $1 \mathrm{D}$ projection of the manifold learning can be in an arbitrary range. This is illustrated in figure 2(a) with exemplary three local signals corresponding to three angular positions. A simple normalization of each of the local signals $\Phi^{j}$ is not sufficient because the extreme positions of the breathing cycle may not 

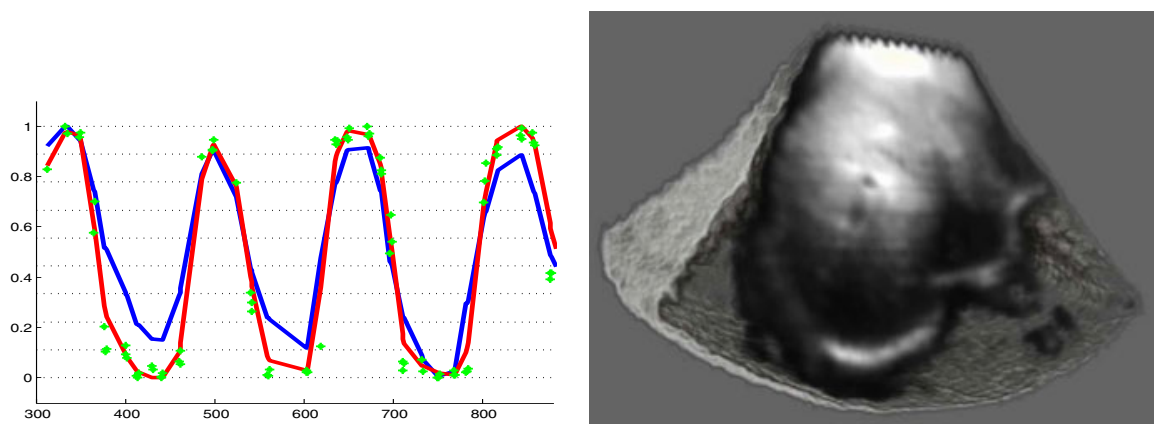

Fig. 3. Aligned local signals (green crosses). Robust spline fitting (red). Ground truth (blue). Dotted lines indicate separation of breathing cycle into several stages. For each stage a volume is compounded, with an exemplary volume rendering on the right image.

be reached within them. Consequently, we affinely register local signals in order to retrieve the best scaling $s^{j}$ and translation $t^{j}$

$$
\Phi^{j} \mapsto s^{j} \cdot \Phi^{j}+t^{j} .
$$

This is, in fact, a groupwise registration scenario, where we choose to align each pair of neighboring curves with a pairwise registration, starting from the middle one. The result of the alignment is shown in figure 2(b)

The values of the partial signals $\Phi^{j}$ are now comparable, however, may still contain outliers. Consequently, we apply a robust curve fitting to all the sample points to retrieve the global breathing signal. We experimented with various curve models, including Fourier, sum of sine waves, and splines. We achieved best results with fitting a spline curve because it allows for the most flexibility, which is important due to irregularity of breathing. The value of the fitted curve then represents the breathing phase of the ultrasound frames.

In a final step, the breathing cycle is classified into several breathing stages. For each of the breathing stages, the ultrasound frames along the various angles are gathered, and compounded into a final volume, see figure 3 .

\section{Experiments}

For our experiments we use the ultrasound system from Ultrasonix (Richmond, Canada) and the optical tracking system from A.R.T. (Weilheim, Germany). Both systems are connected to a workstation PC. For the synchronization, we time stamp the data on the tracking system and use a network time server to calculate the time offset. For the ultrasound data, we use the direct streaming of B-mode images over the network. We perform tests on multiple patient datasets acquired from different positions, focusing on the liver and kidney.

In order to validate our results, we compare them to the measurements of an external gating system. In [13, four different gating systems are compared with 

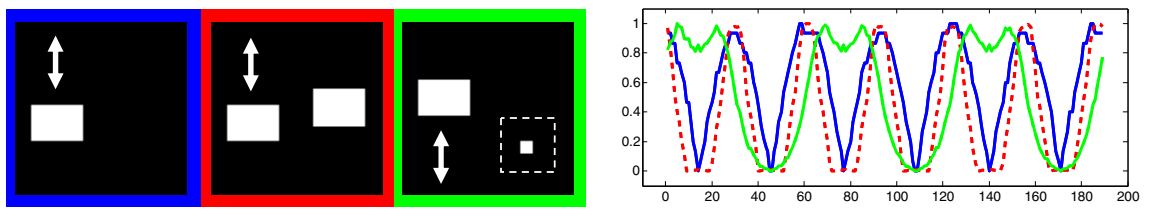

Fig. 4. Analysis of the phase correlation technique for synthetic images. Three different motion scenarios with corresponding energy (gating) curves.

best results for an elastic belt and an optical tracking system. We use the tracking system with markers attached to the chest of the patient. We apply a principal component analysis of the 6D tracking data to find the principal component along whose direction we measure the breathing motion. Further, we low-pass filter the signal to remove cardiac motion and extract the respiratory signal. We refer to the tracked signal as ground truth, which is not completely correct because it contains tracking errors. However, it is the best that can currently be achieved [13] and is sufficient to validate the performance of our image-based approach.

We compare our approach to the phase correlation technique [5]. Unfortunately, we do not achieve meaningful results for our datasets. We think that this is due to the limitation of the technique of approximating the $3 \mathrm{D}$ motion with a global translation in 2D. In order to illustrate this limitation, we produced synthetic images which show periodic motion. The first scenario consists of a rectangle moving up and down, see figure 4. For the second, we add a fixed rectangle, and for the third we add a rectangle that grows and shrinks (see additional material for videos). We plot the corresponding energy curves of the phase correlation technique. For the first scenario (blue) the signal is correct. The addition of a fixed object (red) already leads to a slight distortion, while the addition of the shrinking/growing object (green), leads to an extraction of a false motion signal. Since already the addition of the shrinking/growing object avoids the extraction of the correct motion, it is comprehensible that this approach is not best suited for breathing estimation in a noisy ultrasound environment with $3 \mathrm{D}$ anatomy moving in and out of plane.

The resolution of our ultrasound images is $640 \times 480$ pixels. We downsample the images in each direction by a factor of 2 , leading to $N=\frac{1}{4} \cdot 640 \cdot 480$. This enables a faster processing and leads to no noticeable degradation of the manifold learning. We show excerpts of two data sets in figure 5. We perform all our experiments with a graph neighborhood of $l=14$. The number of images for manifold learning varies between 100 and 300, where we did not notice a dependency of the performance on the number of input samples.

In figure 6, we show the result of the respiratory gating for one of the $2 \mathrm{D}$ datasets together with the ground truth signal. We also calculate the correlation coefficient for multiple 2D data sets, shown in table 1. It is remarkable that the

\footnotetext{
${ }^{1}$ We want to thank the authors of $[5$ for sharing source code.
} 

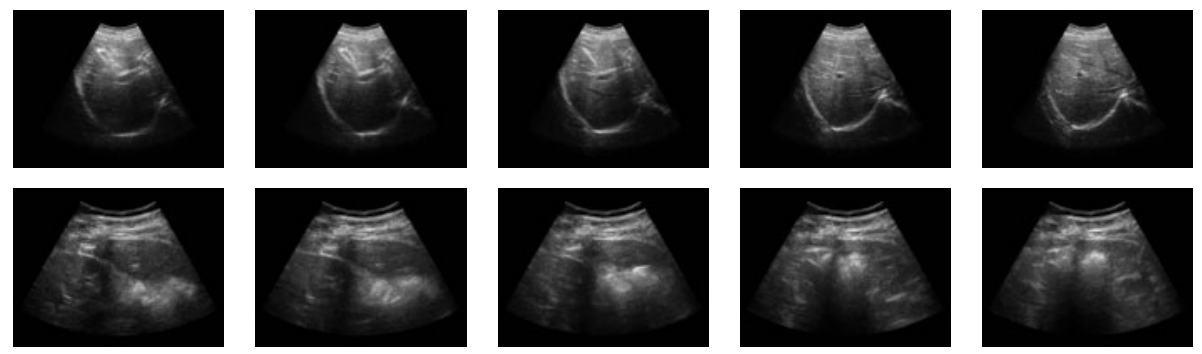

Fig. 5. 2D Ultrasound images over time from liver (abdomen, right upper quadrant, oblique section) and kidney (left lateral decubitus position, right intercostal flank section)

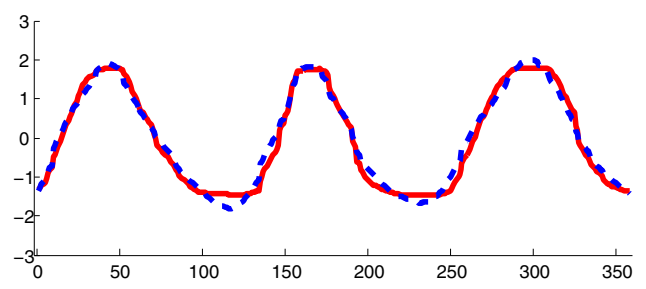

Table 1. 2D

Table 2. 3D

\begin{tabular}{cc}
\hline 2D Correlation \\
\hline liver1 & 95.4 \\
liver2 & 94.4 \\
liver3 & 93.6
\end{tabular}

\begin{tabular}{cc}
\hline 3D Correlation \\
\hline liver $30^{\circ}$ & 94.3 \\
liver $45^{\circ}$ & 95.8 \\
liver $60^{\circ}$ & 96.8 \\
kidney $45^{\circ}$ & 94.4 \\
\hline
\end{tabular}

Fig. 6. Breathing gating results for $2 \mathrm{D}$ (red: estimated signal, blue: ground truth)

kidney $\quad 97.3$

ground truth signal is almost perfectly detected. All peaks in the ground truth signal also appear in the detection. Further, the calculation of the correlation, which is in the range of $95 \%$, confirms the visual similarity of the graphs. We also experimented with normalizing the images before passing them to the manifold learning, noticed however no significant improvement.

For the $4 \mathrm{D}$ experiments, we show the result of a fitted curve in figure 2, We also calculate the correlation coefficient between the fitted curves and ground truth for four datasets, see table 2 . We experimented with three different angular ranges, $30^{\circ}, 45^{\circ}$, and $60^{\circ}$ (maximum of probe), for which the probe steers to 15,21 , and 29 different angular positions. We split the breathing signal into 9 different breathing stages, and compound a 3D volume for each of the stages. A volume rendering of one of the volumes is shown in figure 3 . The additional material contains a video showing the $4 \mathrm{D}$ volume rendering.

All image-based approaches rely on ultrasound acquisitions from the same position, because otherwise it is not possible to differentiate between probe motion and breathing motion. To investigate this assumption, we attached a tracking target to the transducer and analyzed its trajectory. This analysis showed only a negligible deviation. The still position therefore does not limit the applicability of our method, which is also confirmed by our good gating results. 


\section{Conclusion}

We presented an automatic, image-based respiratory gating method for ultrasound using manifold learning. Moreover, we proposed a solution for acquiring $4 \mathrm{D}$ breathing data with a wobbler probe. Our method has the advantage that it is fully automatic and does not require a training phase or prior information about the underlying anatomy. We demonstrated this in our experiments by performing our analysis on various datasets showing different organs and sections. The results of these experiments were very good, for both, 2D and 3D ultrasound data over time. Our approach therefore presents an attractive alternative to external tracking and gating systems with their various setup issues and synchronization problems.

\section{References}

1. Treece, G., Prager, R., Gee, A., Cash, C., Berman, L.: Grey-scale gating for freehand 3D ultrasound. In: ISBI, pp. 993-996 (2002)

2. Zhu, H., Oakeson, K., Friedman, M.: Retrieval of cardiac phase from IVUS sequences. In: Proceedings of SPIE, vol. 5035, p. 135 (2003)

3. de Winter, S., Hamers, R., Degertekin, M., Tanabe, K., Lemos, P., Serruys, P., Roelandt, J., Bruining, N.: A novel retrospective gating method for intracoronary ultrasound images based on image properties. In: Computers in Cardiology (2003)

4. Karadayi, K., Hayashi, T., Kim, Y.: Automatic image-based gating for 4d ultrasound. In: Engineering in Medicine and Biology Society (2006)

5. Sundar, H., Khamene, A., Yatziv, L., Xu, C.: Automatic image-based cardiac and respiratory cycle synchronization and gating of image sequences. In: Yang, G.Z., Hawkes, D., Rueckert, D., Noble, A., Taylor, C. (eds.) MICCAI 2009. LNCS, vol. 5762, pp. 381-388. Springer, Heidelberg (2009)

6. Tenenbaum, J., Silva, V., Langford, J.: A global geometric framework for nonlinear dimensionality reduction. Science 290(5500), 2319 (2000)

7. Belkin, M., Niyogi, P.: Laplacian eigenmaps for dimensionality reduction and data representation. Neural Comput. 15(6) (2003)

8. Zhang, Q., Souvenir, R., Pless, R.: On manifold structure of cardiac MRI data: Application to segmentation. In: CVPR, vol. 1, pp. 1092-1098 (2006)

9. Hamm, J., Davatzikos, C., Verma, R.: Efficient large deformation registration via geodesics on a learned manifold of images. In: Yang, G.-Z., Hawkes, D., Rueckert, D., Noble, A., Taylor, C. (eds.) MICCAI 2009. LNCS, vol. 5761, pp. 680-687. Springer, Heidelberg (2009)

10. Lee, C., Elgammal, A.: Modeling view and posture manifolds for tracking. In: ICCV (2007)

11. Wachinger, C., Mateus, D., Keil, A., Navab, N.: Manifold Learning for Patient Position Detection in MRI. In: ISBI (April 2010)

12. Georg, M., Souvenir, R., Hope, A., Pless, R.: Manifold learning for 4d ct reconstruction of the lung. In: MMBIA (2008)

13. Martinez-Möller, A., Bundschuh, R., Riedel, M., Navab, N., Ziegler, S., Schwaiger, M., Nekolla, S.: Comparison of respiratory sensors and its compliance for respiratory gating in emission tomography. Journal of Nuclear Medicine (2007) 